\section{Bmi-1 is required for maintenance of adult self-renewing haematopoietic stem cells}

\author{
In-kyung Park ${ }^{\star}$, Dalong Qian*, Mark Kiel $\dagger$, Michael W. Becker ${ }^{\star}$, \\ Michael Pihalja ${ }^{\star}$, Irving L. Weissman $\neq$, Sean J. Morrison $\dagger$ \\ \& Michael F. Clarke ${ }^{\star}$
}

* Division of Hematology/Oncology, Internal Medicine, and $\dagger$ Howard Hughes Medical Institute, Department of Internal Medicine, and Department of Cell and Developmental Biology, University of Michigan, Ann Arbor, Michigan 48109, USA

$\ddagger$ Department of Pathology, School of Medicine, Stanford University, Stanford, California 94305, USA

A central issue in stem cell biology is to understand the mechanisms that regulate the self-renewal of haematopoietic stem cells (HSCs), which are required for haematopoiesis to persist for the lifetime of the animal ${ }^{1}$. We found that adult and fetal mouse and adult human HSCs express the proto-oncogene Bmi-1. The number of HSCs in the fetal liver of $\mathrm{Bmi}^{-1} \mathbf{1}^{-1-}$ mice $^{2}$ was normal. In postnatal $\mathrm{Bmi-1}-1-$ mice, the number of HSCs was markedly reduced. Transplanted fetal liver and bone marrow cells obtained from $\mathrm{Bmi}-\mathrm{1}^{-/-}$mice were able to contribute only transiently to haematopoiesis. There was no detectable self-renewal of adult HSCs, indicating a cell autonomous defect in $\mathrm{Bmi}^{-1-1^{-1}}$ mice. A gene expression analysis revealed that the expression of stem cell associated genes ${ }^{3}$, cell survival genes, transcription factors, and genes modulating proliferation including $p 16^{I n k 4 a}$ and $p 19^{A r f}$ was altered in bone marrow cells of the $\mathrm{Bmi-1} 1^{-1-}$ mice. Expression of $p 16^{I n k 4 a}$ and $p 19^{A r f}$ in normal HSCs resulted in proliferative arrest and p53-dependent cell death, respectively. Our results indicate that $\mathrm{Bmi-1}$ is essential for the generation of self-renewing adult HSCs.

To understand the molecular mechanisms that control the selfrenewal of haematopoietic stem cells (HSCs), we have previously constructed a complementary DNA library using highly purified cells with long-term self-renewal potential ${ }^{4}$ and analysed the differential gene expression profile of $\mathrm{HSCs}^{5}$. Analysis of genes expressed in HSCs identified Bmi-1, a member of the Polycomb Group (PcG) family linked to malignant transformation, lymphocyte development, neurological development and senescence of fibroblasts ${ }^{2,6-8}$. Bmi-1 expression, which declines during haematopoietic development ${ }^{9}$, was detected at both single-cell and ten-cell levels by polymerase chain reaction with reverse transcription (RT-PCR) in mouse fetal liver and adult HSCs as well as human normal and leukaemic stem cells, indicating that HSCs express high levels of Bmi-1 (Supplementary Fig. 1).

The Bmi-1 ${ }^{-1-}$ mice develop hypocellular bone marrows and die less than 2 months after birth. To determine the function of Bmi-1 in haematopoiesis, Bmi-1 $1^{-1-}$ mice ${ }^{2}$ in a $\mathrm{C} 57 \mathrm{Bl} / \mathrm{Ka}$. Thy1.1 background $^{1}$ were analysed. As reported previously ${ }^{2}, B m i-1^{-1-}$ mice have normal numbers of peripheral blood myeloid cells but smaller numbers of lymphocytes, whereas the frequency of colony-forming cells in the bone marrow was normal (Supplementary Fig. 2).

In vertebrates, haematopoiesis is organized as a hierarchy, in which HSCs contribute to haematopoiesis for the life of the animal and give rise to transiently reconstituting multipotent progenitors (MPPs) ${ }^{1}$. To determine whether these mice have normal numbers of stem cells that have lost the ability to make progeny or whether they have a defect in the generation of stem cells, we investigated the frequency of HSCs and MPPs from 4-5-week-old Bmi-1 ${ }^{+/+}$, $B m i-1^{+>-}$and $B m i-1^{-1-}$ mice by flow cytometry (Fig. 1a, b). The frequency of HSCs (Thyl.1 ${ }^{\text {low }} \mathrm{c}-\mathrm{kit}^{+} \mathrm{Sca}-1^{+}$Lineage ${ }^{-}$cells) in the bone marrow of heterozygous mice was similar to that of the wildtype littermates (about $0.01 \%$ of bone marrow cells). However, the HSC frequency of the Bmi-1 $1^{-1-}$ mice ranged from $0 \%$ to $0.013 \%$, with an average of $0.005 \%$. When the total numbers of bone marrow cells were taken into account, $B m i-1^{-l-}$ mice had an average 10 -fold (up to 45 -fold) less total HSCs as measured by flow cytometry $(P=0.007$; Supplementary Fig. 3$)$. The frequency of MPP cells (Thy $1.1^{\text {low }} \mathrm{c}-\mathrm{kit}^{+} \mathrm{Sca}-1^{+}$Lineage ${ }^{-/ \text {low }}$ cells, which contain both HSCs and a majority population of MPPs) in the $B m i-1^{-1-}$ bone marrow was similar to that of wild-type mice (about $0.1 \%$ of bone marrow cells). Next, competitive repopulation experiments ${ }^{1}$ were performed to determine the long-term reconstituting abilities of bone marrow mononuclear cells obtained from 5-week-old $B m i-1^{-l-}$ mice. At 5 weeks after transplantation, flow cytometry analysis of peripheral blood revealed that bone marrow cells from both wild-type and heterozygous mice efficiently reconstituted myeloid cells, B cells and T cells, whereas Bmi-1 ${ }^{-1-}$ bone marrow cells showed a decreased capacity to reconstitute all lineages in the transplanted animals (Fig. 1c). By 10 weeks, the mice reconstituted with $B m i-1^{-1-}$ marrow, but not with wild-type bone marrow, lost nearly all donor-derived mature haematopoietic cells (Fig. 1d). These results indicate that $\mathrm{Bmi}-1$ might be required for self-renewal and/or maintenance of adult HSCs.

The defect in HSCs in the bone marrow of $B m i-1^{-1-}$ mice could result from an impaired generation of self-renewing fetal and/or adult HSCs, defective stem cell homing, or a defective bone marrow microenvironment. We therefore next examined the HSC compartment in fetal Bmi-1 $1^{-1-}$ mice. As measured by flow cytometry, Bmi-1 ${ }^{-1-}$ mice had similar numbers of fetal liver cells and an almost identical frequency of fetal liver HSCs ${ }^{10}$ (Thyl. $1^{\text {low }} \mathrm{Mac}-1^{+} \mathrm{Sca}-1^{+}$Lineage ${ }^{-}$) as their wild-type littermates (Fig. 2a). Furthermore, the fetal liver HSCs from $\mathrm{Bmi}^{-1} \mathrm{1}^{-1-}$ mice migrated normally towards the chemokine SDF- $1 \alpha$, and fetal liver cells expressed normal levels of SDF-1 $\alpha$ and its receptor CXCR4, important mediators of HSC homing ${ }^{11}$ (Supplementary Fig. 4).
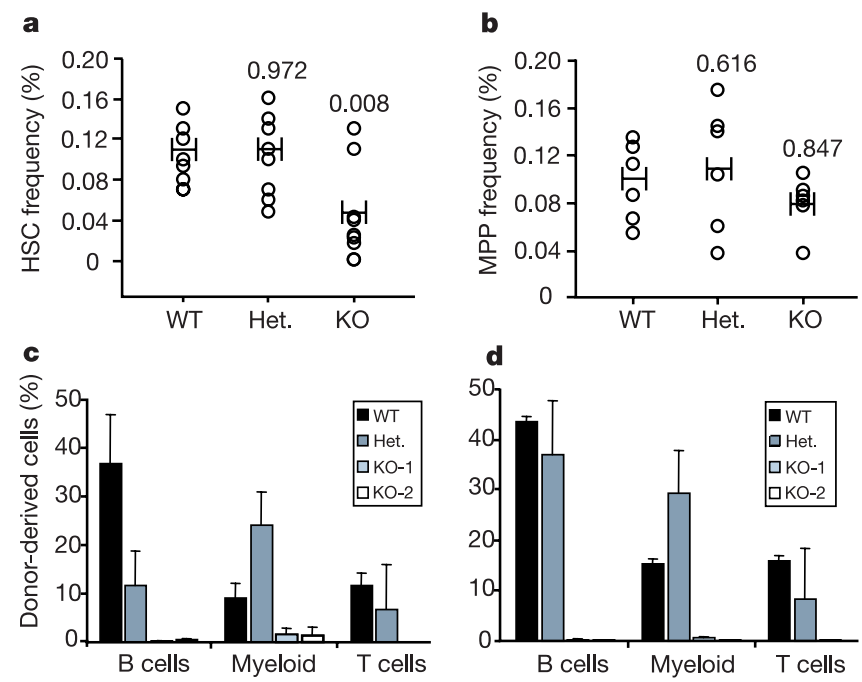

Figure 1 Analysis of adult HSCs. $\mathbf{a}, \mathbf{b}$, Effect of Bmi-1 deletion on the frequencies of HSCs and MPPs. The bars show the average frequencies of HSCs $(\mathbf{a})$ and MPPs $(\mathbf{b})$ from eight individual mice in each group. $P$ values (shown above the bars) were calculated with the unpaired Student's $t$-test. WT, wild type; Het., $\mathrm{Bmi}^{-1^{+/-}} ; \mathrm{KO}, \mathrm{Bmi-} 1^{-1-}$. c, d, Competitive reconstitution. Donor (Ly5.1) bone marrow cells $\left(5 \times 10^{5}\right)$ were mixed with the same number of Ly5.2 bone marrow cells and injected into lethally irradiated Ly5.2 mice $(n=2-4)$. Peripheral blood was analysed 5 weeks (c) and 10 weeks (d) after reconstitution for donor-derived myeloid, B-lymphoid and T-lymphoid cells. KO-1 and $\mathrm{KO}-2, \mathrm{Bmi}^{-1}{ }^{-1-}$ mice 1 and 2. 
Although it is possible that Bmi-1 affects homing by other pathways, these data suggest that the HSC defect in $B m i-1^{-1-}$ bone marrow is not likely to be the result of defective fetal liver HSC migration to the bone marrow.

If the defects in the Bmi-1 $1^{-1-}$ mice were secondary to an abnormal microenvironment, then fetal liver stem cells from these mice would be able to engraft the bone marrow of wild-type mice efficiently. If the defects were in the generation of self-renewing HSCs, then $B m i-1^{-1-}$ fetal liver HSCs would fail to permanently engraft the bone marrow of normal adult mice. To distinguish between these possibilities, lethally irradiated Ly5.2 congenic mice were transplanted with $5 \times 10^{5}, 10^{6}$ and $5 \times 10^{6} \mathrm{Bmi}^{-1} \mathrm{1}^{-1-}$ cells, or $5 \times 10^{5}$ and $10^{6} \mathrm{Bmi}^{-1++}$ embryonic day 14.5 (E14.5) fetal liver cells. In addition, competitive reconstitution assays were performed with $10^{6}$ fetal liver cells from $B m i-1^{+/+}$or $B m i-1^{-1-}$ mice mixed with $10^{6}$ Ly5.2 bone marrow cells. At 4 weeks after transplantation, Bmi-1 $1^{-1-}$ mice showed reconstitution of $\mathrm{T}$ cells and myeloid cells, whereas B-cell reconstitution was greatly decreased (Fig. 2b). By 8 weeks, virtually no donor-derived cells were detected in recipient bone marrows reconstituted with $B m i-1^{-l-}$ fetal liver cells (Fig. 2c), suggesting a severe defect in the HSCs' ability to maintain haematopoiesis. At 8 weeks after the original transplant, secondary bone marrow transplants were performed with $10^{7}$ donor cells. Peripheral blood analysis after 6 weeks indicated that there were no detectable $B m i-1^{-1-}$ cells in the mice with secondary transplants (Fig. 2d). Taken together with

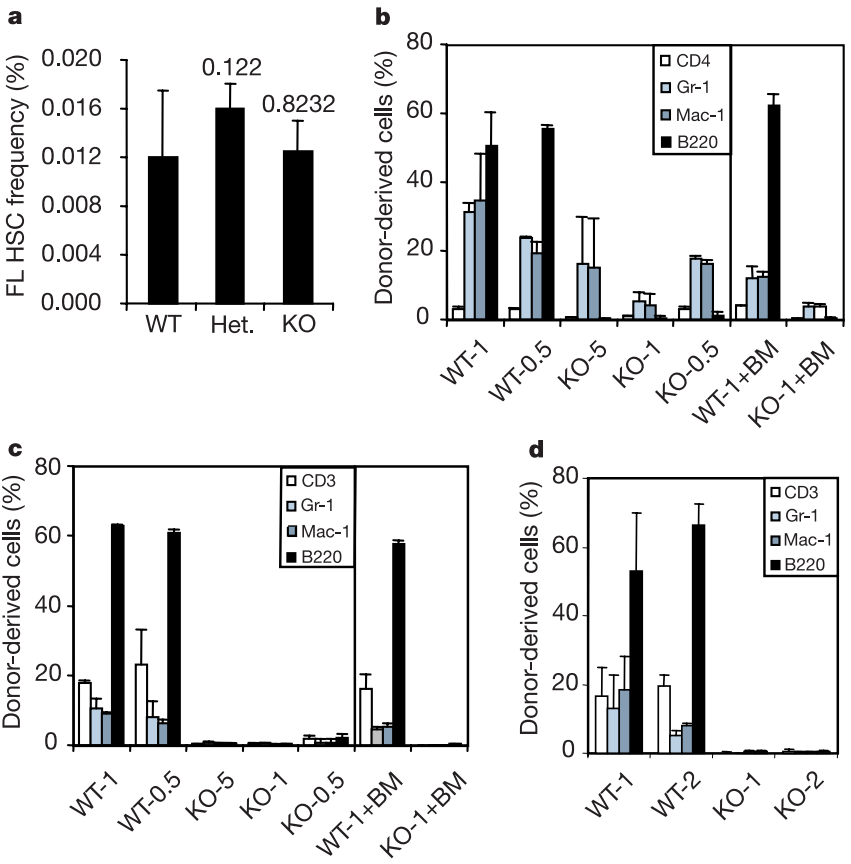

Figure 2 Analysis of fetal liver haematopoietic stem cells in $\mathrm{Bmi-} \mathrm{1}^{+/+}, \mathrm{Bmi}-1^{+/-}$and $B \mathrm{mi}^{-} 1^{-/-}$mice. $\mathbf{a}$, The fetal liver HSC population was analysed from two $B \mathrm{mi}^{-1^{+/+}}$(WT), three $B \mathrm{mi}^{-1^{+/-}}$(Het.) and four Bmi-1 ${ }^{-/-}$(KO) fetuses by flow cytometry. $P$ values (shown above the bars) were calculated with the unpaired Student's $t$-test

b, c, Peripheral blood analysis of lethally irradiated mice reconstituted with $10^{6}$ and $5 \times 10^{5} \mathrm{Bmi}^{-1} 1^{+/+}$fetal liver cells (WT-1 and WT-0.5, respectively) and $5 \times 10^{6}, 10^{6}$ and $5 \times 10^{5} \mathrm{Bmi}_{-1}{ }^{-1-}$ fetal liver cells (K0-5, KO-1 and K0-0.5, respectively). WT-1 + BM and $\mathrm{KO}-1+\mathrm{BM}$ indicate competitive reconstitution with $10^{6}$ fetal liver cells together with $10^{6}$ Ly5.2 bone marrow cells (five mice in each group). Data for 4 weeks (b) and 8 weeks (c) after reconstitution are shown. $\mathbf{d}$, Secondary bone marrow transfer. After 8 weeks of reconstitution, $10^{7}$ bone marrow cells from mice non-competitively reconstituted with $10^{6}$ fetal liver cells were used to transplant lethally irradiated Ly5.2 mice. Peripheral blood analysis was performed 6 weeks after transplant. the marked decrease in HSC numbers in the bone marrow of the $B m i-1^{-1-}$ mice, these results demonstrate that there is a profound intrinsic defect in the maintenance of self-renewing HSCs in the mutant mice.

To begin to understand the molecular mechanism by which Bmi-1 affects the generation of HSCs, we compared the gene expression profiles of bone marrow mononuclear cells obtained from wild-type and Bmi-1 ${ }^{-/-}$mice. Bmi-1 is a member of the $P c G$ family of transcriptional repressors that control development by the regulation of cell growth and differentiation genes ${ }^{12}$. Microarray analysis with bone marrow RNAs isolated from wild-type and $B m i-1^{-l-}$ mice identified several interesting genes (Fig. 3a, b and Supplementary Table 2). These include wild-type p53-induced gene 1 (Wig1), tight-junction protein 1 (TJP1) and platelet-activating factor acetylhydrolase isoform $1 \mathrm{~b}(\mathrm{PAF}-\mathrm{AH} \gamma)$, all of which are expressed in embryonic, neuronal and haematopoietic stem cells ${ }^{3}$, as well as several transcription factors, signalling proteins and apoptosis inhibitor 6 . As reported previously in embryonic fibroblasts, expression of $p 16^{\text {Ink4a }}$ and $p 19^{\text {Arf }}$, which are generated by alternative splicing from the Ink $4 a$ locus ${ }^{13-15}$, in adult bone marrow of $B m i-1^{-/-}$mice was elevated compared with wild-type littermates (Fig. 3a). A complete list of differentially expressed genes is given in Supplementary Table 3 . In contrast with the analysis of the adult bone marrow, a microarray analysis of E14.5 fetal liver cells did not show significant differences in the gene expression by the wild-type and mutant cells (data not shown), which is consistent with the observation that Bmi-1 is not crucial for fetal liver haematopoiesis. Some members of the Hox family are also thought to be targets of Bmi-1. Semiquantitative RT-PCR indicated that expression of Hoxb4, which is important for HSC development ${ }^{16}$, was not altered in Bmi-1 ${ }^{-1-}$ mice whereas Hoxa9, which functions in cell fate decisions of haematopoietic progenitors ${ }^{17}$, was slightly upregulated in thymus and bone marrow of $B m i-1^{-l-}$ mice (Supplementary Fig. 5).

Next, the effects of the Bmi-1 targets, $p 16^{\text {Ink4a }}$ and $p 19^{\text {Arf }}$, on HSC function were determined. HSCs $\left(\mathrm{c}-\mathrm{Kit}^{+} \mathrm{Sca}-1^{+} \mathrm{Flt} 3^{-}\right.$(Flk-2) Lineage $^{-}$phenotype $\left.{ }^{18}\right)$ were isolated and infected with mouse stem cell virus (MSCV) expressing green fluorescent protein (GFP) alone or together with $p 16^{\text {Ink } 4 a}$ or $p 19^{A r f}$. Two days after infection, only $6 \%$ of cells infected with the control virus and the $p 16^{\text {Ink4a }}$ virus, compared with $60 \%$ of the cells infected with the $p 19^{\text {Arf }}$ virus, had lost viability as measured by exclusion of a viability dye 7AAD (Fig. 4). When single viable GFP-positive HSCs were placed in culture medium, $79 \%$ of the haematopoietic progenitors infected with the control GFP virus generated more than 50 cells after 7 days of culture (75 of 95 wells seeded). By contrast, progenitors infected with the $p 16^{\text {Ink } 4 a}$ virus either did not prolifer-

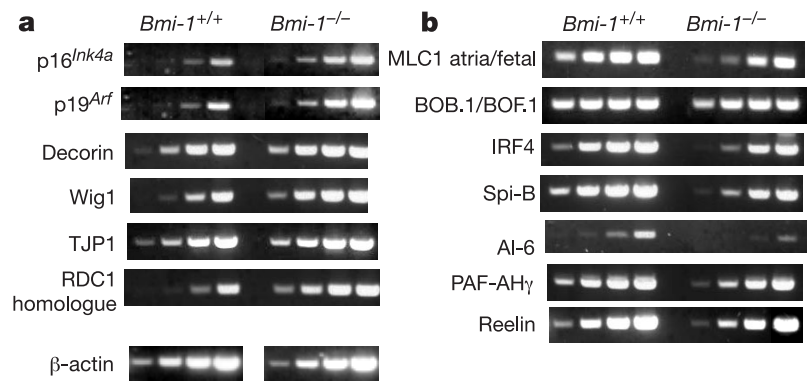

Figure 3 Regulation of gene expression by Bmi-1. Semiquantitative RT-PCR of genes upregulated (a) and downregulated (b) in $B m i-1^{-1-}(\mathrm{KO})$ mice. cDNA was reverse transcribed from $2 \mu \mathrm{g}$ of total RNA, and gene-specific primers were used for PCR. Aliquots were taken at the end of the 26th, 29th, 32nd and 35th cycles. The $\beta$-actin aliquots were taken at the end of the 17th, 20th, 23rd and 26th cycles. 


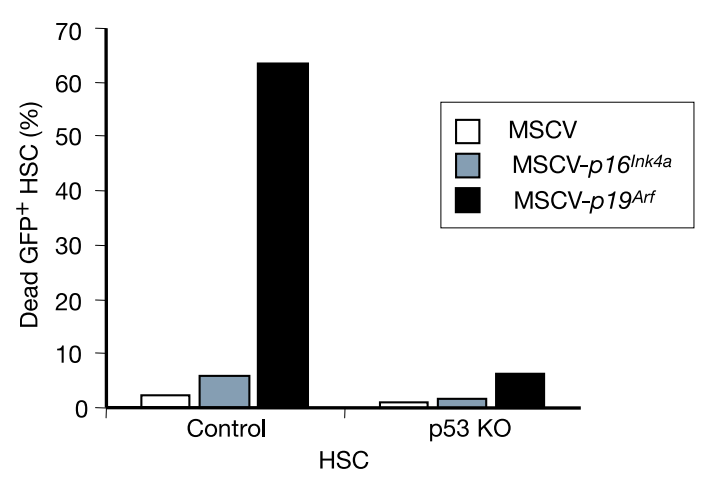

Figure 4 Effects of $p 16^{\text {Ink4a }}$ and $p 19^{\text {Arf }}$ on HSC proliferation. HSCs from wild-type and $p 53^{-1-}(\mathrm{KO})$ mice were infected with various MSCV viruses. After $48 \mathrm{~h}$, cells were analysed for GFP expression by flow cytometry. Percentages of GFP-positive 7AADpositive (dead transduced) cells are shown.

ate at all or generated only a few cells (3-10 cells, in 3 of 95 wells). None of the $\mathrm{p} 19^{\text {Arf }}$ virus-infected HSCs proliferated ( 0 of 47 wells). The $\mathrm{p} 19^{\text {Arf }}$ protein increases the level of p53 protein ${ }^{19}$. To determine whether $p 19^{A r f}$-induced cell death is mediated by $\mathrm{p} 53$, we infected p $53^{-1-}$ HSCs (c-Kit ${ }^{+} \mathrm{Sca}-1^{+} \mathrm{Flt} 3^{-}$Lineage ${ }^{-}$phenotype) with the p1 $19^{\text {Arf }}$ virus. In contrast with the wild-type HSCs, these cells were viable 2 days after infection (Fig. 4). These results suggest that Bmi-1 targets $p 16^{\text {Ink } 4 a}$ to allow the proliferation of HSCs and $p 19^{\text {Arf }}$ to inhibit apoptosis through p53.

HSCs undergo self-renewing cell divisions for the lifetime of an animal. Once an HSC has undergone cell division, the daughter cells must choose one of three possible fates: to remain as a stem cell, to differentiate into progenitors or to undergo apoptosis (Fig. 5). Although several genes have been shown to be important for stem cell function, the mechanisms that regulate the self-renewal of HSCs are poorly understood ${ }^{20-26}$. Our results suggest that Bmi-1 is necessary for efficient self-renewing cell divisions of adult HSCs but is less critical for the generation of differentiated progeny (Fig. 5). Transplantation of Bmi-1 $1^{-1-}$ fetal liver cells resulted in transient haematopoietic cell reconstitution. This indicates that the transplanted mutant fetal liver HSCs might not have generated HSCs efficiently but might have given rise to MPPs that could sustain haematopoiesis for only $4-8$ weeks. This could have been due to the expression of other PcG genes by the MPPs. Consistent with this model was our previous result that MPPs express more Enx-1 than do HSCs ${ }^{5}$. Similarly, other $P c G$ genes, such as rae28, whose expression is necessary for fetal liver haematopoiesis to occur $^{27}$, might permit HSC proliferation in the fetus of the mutant mice. This is consistent with the observation that the expression of p $19^{\text {Arf }}$ is elevated in adult bone marrow cells, but not in fetal liver cells, of the mutant mice (Supplementary Fig. 5). Alternatively, the number of self-renewing cell division of $B m i-1^{-1-}$ FL-HSCs might be restricted.

The mechanism by which Bmi-1 modulates HSC self-renewal seems to be through the regulation of genes important for stem cell fate decisions as well as that of survival genes, anti-proliferative genes and stem-cell-associated genes. The gene expression data and retroviral infection experiments implicate $p 16^{\text {Ink } 4 a}, p 19^{\text {Arf }}$ and p53 as downstream effectors of Bmi-1 involved in the control of the proliferation and survival of HSCs during self-renewing cell divisions (Fig. 5). However, owing to the paucity of HSCs in the $B m i-1^{-1-}$ mice, it is not possible to determine whether the expression levels of these genes are comparable to those of HSCs infected with the $p 16^{\operatorname{Ink} 4 a}$ and $p 19^{A r f}$ retroviruses. Although the increased expression of the p53-target gene Wig1 suggests that p19 $9^{\text {Arf }}$ is activated in Bmi-1 $1^{-1-}$ haematopoietic cells, the relative contribution of each of these pathways to the regulation of selfrenewing HSC generation awaits careful analysis of the HSCs of
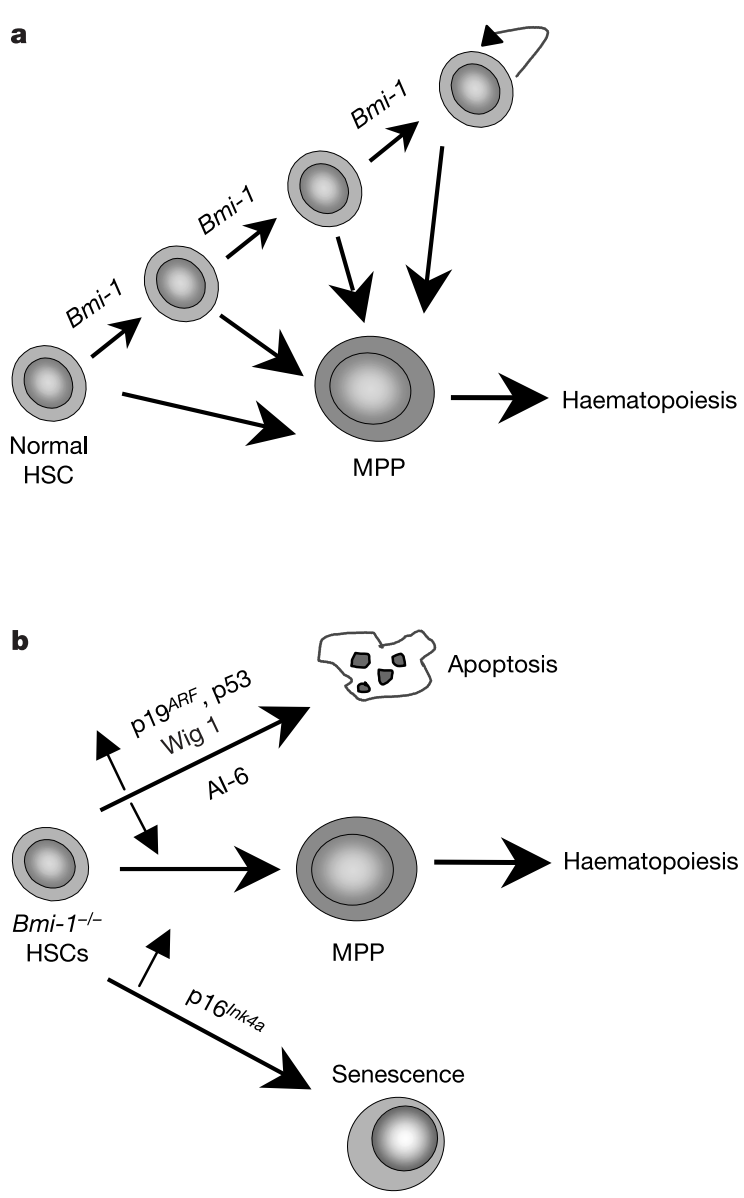

Figure 5 Proposed model for the role of Bmi-1 in HSC maintenance. a, In wild-type mice, a HSC can undergo asymmetric cell division to generate another HSC as well as a MPP, thereby maintaining the HSC pool. $\mathbf{b}$, In the absence of Bmi-1, the increased expression of $p 16^{\operatorname{lnk} 4 a}$ can induce a proliferative arrest and increased expression of $p 19^{\text {Arf }}$ can increase the level of p53 and its downstream target, Wig1, which, coupled with a decreased expression of apoptosis inhibitor 6 (Al-6), results in the death of HSCs. This results in severely impaired adult HSC self-renewal. By contrast, MPPs, which are initially generated from the HSCs, transiently give rise to mature haematopoietic cells.

$B m i-1^{-l-}$ mice that also harbour mutations of one or more of these other genes. Some of the other genes identified in the microarray analysis might also have crucial roles in stem cell biology. For example, Wig1 and apoptosis inhibitor 6 might regulate the survival of HSCs. As Bmi-1 has now been shown ${ }^{30}$ to be necessary for the maintenance of mouse leukaemia and Bmi-1 is expressed by human AML stem cells, it will be of interest to determine whether Bmi-1 has a similar function in human leukaemia.

\section{Methods}

\section{Flow cytometry}

Bone marrow cells were obtained by flushing the tibias and femurs of mice, and stained with antibodies against lineage markers (CD3, CD4, CD5, CD8, Gr-1, Mac-1 and Ter119), Sca-1, c-Kit and Thyl.1 as described ${ }^{1}$. Cells were analysed by flow cytometry with a Vantage fluorescence-activated cell sorter (Becton Dickinson). The phenotype of adult HSCs, multipotent progenitors (MPPs) and fetal liver HSCs are Thyl. $1^{\text {low }_{\mathrm{C}}-\mathrm{Kit}^{+}} \mathrm{Sca}-$

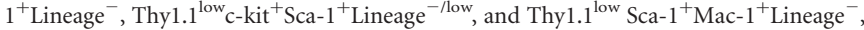
respectively. When Thy1.2 background mice (C57BL/6J p53-deficient mice and C57BL/6J mice) were used, HSCs and MPPs were isolated as c- $\mathrm{Kit}^{+} \mathrm{Sca}-{ }^{+}{ }^{+} \mathrm{Flt} 3^{-}$Lineage $^{-}$and c-Kit ${ }^{+}$Sca- $1^{+}$Flt $3^{+}$Lineage ${ }^{-}$cells, respectively ${ }^{18}$.

\section{Competitive reconstitution}

Lethally irradiated $\mathrm{C} 57 \mathrm{Bl} / \mathrm{Ka}-\mathrm{Ly} 5.2$ congenic mice were competitively reconstituted with whole bone marrow or E14.5 fetal liver cells from Bmi-1 ${ }^{+/+}, \mathrm{Bmi}_{-1}^{+/-}$and $\mathrm{Bmi-1}{ }^{-1-}$ 
mice with or without the C57Bl/Ka-Ly5.2 recipient bone marrow cells ${ }^{1}$. Reconstitution of donor (Ly5.1) myeloid and lymphoid cells was monitored by staining blood cells with antibodies against Ly5.1, CD3, B220, Mac-1 and Gr-1. The secondary bone marrow transplant was performed with $10^{7}$ whole bone marrow cells from mice reconstituted with $B m i-1^{+/+}$or $B m i-1^{-1-}$ fetal liver cells.

\section{Retroviral gene transfer of HSCs}

Mouse stem cell viruses expressing mouse $p 16^{\text {Ink } 4 a}$ or $p 19^{A r f}$ cDNAs together with GFP were produced using Phoenix ecotropic packaging cells ${ }^{28}$. Infection of HSCs was done as described ${ }^{29}$ except that three cycles of infections were performed. After $48 \mathrm{~h}$, single GFP-positive cells were sorted into a 96-well plate containing $100 \mu \mathrm{l} \mathrm{HSC} \mathrm{medium}{ }^{29}$ and grown for 7 days. Each well was scored for the presence of GFP-positive cells by observation with a fluorescence microscope.

Received 10 February; accepted 19 March 2003; doi:10.1038/nature01587.

Published online 20 April 2003.

1. Morrison, S. J. \& Weissman, I. L. The long-term repopulating subset of hematopoietic stem cells is deterministic and isolatable by phenotype. Immunity 1, 661-673 (1994).

2. van der Lugt, N. M. et al. Posterior transformation, neurological abnormalities, and severe hematopoietic defects in mice with a targeted deletion of the bmi-1 proto-oncogene. Genes Dev. 8 , 757-769 (1994).

3. Ramalho-Santos, M. et al. 'Stemness': transcriptional profiling of embryonic and adult stem cells. Science 298, 597-600 (2002)

4. Park, I.-K. et al. Molecular cloning and characterization of a novel regulator of G-protein signaling from mouse hematopoietic stem cells. J. Biol. Chem. 276, 915-923 (2001).

5. Park, I. K. et al. Differential gene expression profiling of adult murine hematopoietic stem cells. Blood 99, 488-498 (2002).

6. Lessard, J., Baban, S. \& Sauvageau, G. Stage-specific expression of Polycomb group genes in human bone marrow cells. Blood 91, 1216-1224 (1999).

. Kiyono, T. et al. Both $\mathrm{Rb} / \mathrm{p}^{1} 6^{\mathrm{INK} 4 \mathrm{a}}$ inactivation and telomerase activity are required to immortalize human epithelial cells. Nature 396, 84-88 (1998).

8. van der Lugt, N. M. T., Alkema, M., Berns, A. \& Deschamps, J. The Polycomb-group homolog Bmi-1 is a regulator of murine Hox gene expression. Mech. Dev. 58, 153-164 (1996).

9. Akashi, K. et al. Transcriptional accessibility for genes of multiple tissues and hematopoietic lineages is hierarchically controlled during early hematopoiesis. Blood 101, 383-389 (2003).

10. Morrison, S., Hemmati, H., Wandycz, A. \& Weissman, I. The purification and characterization of fetal liver hematopoietic stem cells. Proc. Natl Acad. Sci. USA 92, 10302-10306 (1995).

11. Wright, D. E. et al. Hematopoietic stem cells are uniquely selective in their migratory reponse to chemokines. J. Exp. Med. 195, 1145-1154 (2002).

12. Mahmoudi, T. \& Verrijzer, C. P. Chromatin silencing and activation by Polycomb and trithorax group proteins. Oncogene 20, 3055-3066 (2001).

13. Weber, J. D. et al. Nucleolar Arf sequesters Mdm2 and activates p53. Nature Cell Biol. 1, 20-26 (1999).

14. Jacob, J. et al. The oncogene and Polycomb-group gene bmi-1 regulates cell proliferation and senescence through the ink4a locus. Nature 397, 164-168 (1999).

15. Quelle, D. E., Zindy, F., Ashmun, R. A. \& Sherr, C. J. Alternative reading frames of the INK4a tumour suppressor gene encode two unrelated proteins capable of inducing cell cycle arrest. Cell 84, 993-1000 (1995).

16. Antonchuk, J., Sauvageau, G. \& Humphries, R. K. HOXB4 overexpression mediates very rapid stem cell regeneration and competitive hematopoietic repopulation. Exp. Hematol. 29, 1125-1134 (2002).

17. Lawrence, H. J. et al. Mice bearing a targeted interruption of the homeobox gene HOXA9 have defects in myeloid, erythroid, and lymphoid hematopoiesis. Blood 89, 1922-1930 (1997).

18. Christensen, J. L. \& Weissman, I. L. Flk-2 is a marker in hematopoietic stem cell differentiation: a simple method to isolate long-term stem cells. Proc. Natl Acad. Sci. USA 98, 14541-14546 (2001)

19. Zhang, Y., Xiong, Y. \& Yarbrough, W. G. ARF promotes MDM2 degradation and stabilizes p53: ARF-INK4a locus deletion impairs both the Rb and p53 tumour suppression pathways. Cell 92, 725-734 (1998)

20. Shivdasani, R., Mayer, E. \& Orkin, S. Absence of blood formation in mice lacking the T-cell leukaemia oncoprotein tal-1/SCL. Nature 373, 432-434 (1995).

21. Porcher, C. et al. The T cell leukemia oncoprotein SCL/tal-1 is essential for development of all hematopoietic lineages. Cell 86, 47-57 (1996).

22. Antonchuk, J., Sauvageau, G. \& Humphries, R. K. HOXB4-induced expansion of adult hematopoietic stem cells ex vivo. Cell 109, 39-45 (2002).

23. Domen, J., Cheshier, S. H. \& Weissman, I. L. The role of apoptosis in the regulation of hematopoietic stem cells: overexpression of BCL-2 increases both their number and repopulation potential. J. Exp. Med. 191, 253-264 (2000)

24. Nichogiannopoulou, A. et al. Defects in hemopoietic stem cell activity in Ikaros mutant mice. J. Exp. Med. 190, 1201-1214 (1999).

25. Fisher, R. C., Lovelock, J. D. \& Scott, E. W. A critical role for PU.1 in homing and long-term engraftment by hematopoietic stem cells in the bone marrow. Blood 94, 1283-1290 (1999).

26. Cheng, T. et al. Hematopoietic stem cell quiescence maintained by $\mathrm{p} 21^{\text {cip } 1 / \text { wafl }}$. Science $\mathbf{2 8 7}, 1804-1808$ (2000).

27. Ohta, H. et al. Polycomb group gene rae28 is required for sustaining activity of hematopoietic stem cells. J. Exp. Med. 195, 759-770 (2002).

28. Pear, W., Nolan, G., Scott, M. \& Baltimore, D. Production of high-titer helper-free retroviruses by transient transfection. Proc. Natl Acad. Sci. U.S.A. 90, 8392-8396 (1993).

29. Cotta, C., Swindle, C., Weissman, I. L. \& Klug, C. A. Retroviral Transduction of FACS-Purified Hematopoietic Stem Cells (eds Klug, C. A. \& Jordan, C. T.) 243-252 (Humana Press, Totowa, New Jersey, 2001).

30. Lessard, J. \& Sauvageau, G. Bmi-1 determines the proliferative capacity of normal and leukaemic stem cells. Nature advance online publication, 20 April 2003 (doi: 10.1038/nature01572).

Supplementary Information accompanies the paper on www.nature.com/nature.
Acknowledgements We thank T. Magnuson and C. Klug for providing $B m i-1^{+/-}$mice and the MSCV plasmid, respectively; and the Flow Cytometry Core and the Microarray Core at the University of Michigan for their work. The Microarray Core is supported in part by a University of Michigan's Cancer Center Support Grant from the NIH. This work is supported by grants from the NIH.

Competing interests statement The authors declare that they have no competing financial interests.

Correspondence and requests for materials should be addressed to M.F.C. (mclarke@umich.edu).

\section{DNA helicase Srs2 disrupts the Rad51 presynaptic filament}

\section{Lumir Krejci ${ }^{\star}$, Stephen Van Komen ${ }^{\star} \dagger$, Ying Lił, Jana Villemain ${ }^{\star}$, Mothe Sreedhar Reddy ${ }^{\star}$, Hannah Klein $\$, Thomas Ellenberger $\ddagger$ \& Patrick Sung ${ }^{\star}$}

* Institute of Biotechnology and Department of Molecular Medicine, University of Texas Health Science Center at San Antonio, 15355 Lambda Drive, San Antonio, Texas 78245, USA

$\ddagger$ Department of Biological Chemistry and Molecular Pharmacology, Harvard Medical School, Boston, Massachusetts 02115, USA

$\$$ Department of Biochemistry, New York University School of Medicine, New York, New York 10016, USA

$\dagger$ These authors contributed equally to the work

Mutations in the Saccharomyces cerevisiae gene SRS2 result in the yeast's sensitivity to genotoxic agents, failure to recover or adapt from DNA damage checkpoint-mediated cell cycle arrest, slow growth, chromosome loss, and hyper-recombination ${ }^{1,2}$. Furthermore, double mutant strains, with mutations in DNA helicase genes $S R S 2$ and $S G S 1$, show low viability that can be overcome by inactivating recombination, implying that untimely recombination is the cause of growth impairment ${ }^{1,3,4}$. Here we clarify the role of $S R S 2$ in recombination modulation by purifying its encoded product and examining its interactions with the Rad51 recombinase. Srs2 has a robust ATPase activity that is dependent on single-stranded DNA (ssDNA) and binds Rad51, but the addition of a catalytic quantity of Srs2 to Rad51-mediated recombination reactions causes severe inhibition of these reactions. We show that Srs 2 acts by dislodging Rad51 from ssDNA. Thus, the attenuation of recombination efficiency by Srs2 stems primarily from its ability to dismantle the Rad51 presynaptic filament efficiently. Our findings have implications for the basis of Bloom's and Werner's syndromes, which are caused by mutations in DNA helicases and are characterized by increased frequencies of recombination and a predisposition to cancers and accelerated ageing ${ }^{5}$.

We have been unable to overexpress Srs2 protein significantly in yeast, suggesting that this protein is unstable in, and/or toxic to, yeast cells. We therefore turned to Escherichia coli and an inducible T7 promoter as vehicle for Srs 2 expression. Srs 2 could be revealed by Coomassie Blue staining of $E$. coli extracts and by immunoblotting with antibodies against Srs2 (Fig. 1a). We subjected E. coli lysate to precipitation with ammonium sulphate and a five-step chromatographic fractionation scheme to purify Srs2 to near-homogeneity (Fig. 1b). Purified Srs2 has a robust ssDNA-dependent ATPase activity $\left(k_{\text {cat }} \geq 2,500 \mathrm{~min}^{-1}\right)$ and a DNA helicase activity ${ }^{6}$ that is fuelled by ATP hydrolysis (Fig. 1c).

Previous studies have unveiled an anti-recombination function in SRS2 and a genetic interaction with RAD51 (refs 7-9). We investigated whether Srs2 protein interacts physically with Rad51 protein, and also tested its effect on the Rad51 recombinase activity ${ }^{10}$. To 